DOI: $10.20472 /$ IAC.2018.043.004

\author{
MARÍA J. BLANCA \\ University of Malaga and University of Barcelona, Spain
}

ROSER BONO

University of Barcelona, Spain

JAUME ARNAU

Department of Social Psychology and Quantitative Psychology, University of Barcelona, Spain

RAFAEL ALARCÓN

Department of Psychobiology and Behavioural Sciences Methodology, University of Malaga, Spain

JUANA GÓMEZ-BENITO

Department of Social Psychology and Quantitative Psychology, University of Barcelona, Spain

\title{
WHICH ARE THE MOST COMMON DISTRIBUTIONS IN SOCIAL, HEALTH, AND EDUCATION SCIENCES?
}

\begin{abstract}
:
Statistical analysis is crucial for research and the choice of analytical technique should take into account the specific distribution of data. Although the data obtained from health, educational and social sciences research are often not normally distributed, there are very few studies detailing which distributions are most likely to represent data in these disciplines. The aim of the present study was to determine the frequency of appearance of the most common non-normal distributions in the health, educational and social sciences by means of a systematic review. The search was carried out in the Web of Science (WOS) database, from which we retrieved 984 abstracts of papers published between 2010 and 2015. In the final review, 148 papers from the area of health, 18 from education and 96 from the social sciences were included. The selection was performed independently by two reviewers. The inter-rater reliability for article selection and agreement regarding the type of distribution was high. The results showed that distributions from the exponential family are the most common non-normal distributions - and more specifically, gamma as a continuous distribution and the negative binomial as a discrete distribution. In addition to identifying the most common distributions for real data these results will help researchers to decide which distributions should be used in simulation studies examining statistical procedures.

This research was supported by grant PSI2016-78737-P (AEI/FEDER, UE) from the Spanish Ministry of Economy, Industry and Competitiveness.
\end{abstract}

\section{Keywords:}

non-normal distributions, exponential family distributions, systematic review.

JEL Classification: C46, C15, C18 